\title{
Meeting high standards: the effect of perfectionism on task performance, self-esteem, and self-efficacy in college students
}

\author{
Brandy M. Chufar, Terry F. Pettijohn II \\ Department of Psychology, Coastal Carolina University, Conway, South Carolina, USA
}

Email address:

bmchufar@coastal.edu (B. Chufar), pettijohn@coastal.edu (T. F. Pettijohn II)

\section{To cite this article:}

Brandy M. Chufar, Terry F. Pettijohn II. Meeting High Standards: The Effect of Perfectionism on Task Performance, Self-Esteem, and Self-Efficacy in College Students. Psychology and Behavioral Sciences. Vol. 2, No. 3, 2013, pp. 117-123.

doi: $10.11648 /$ j.pbs.20130203.16

\begin{abstract}
Perfectionism is currently believed to be a multidimensional construct. This study focused on the perspective that perfectionism has both positive and negative qualities. Specifically, this study predicted that adaptive perfectionists would perform better on a task and have higher levels of self-esteem and self-efficacy than maladaptive perfectionists. Maladaptive perfectionists, in general, have been found to have lower performance, self-esteem, and self-efficacy. Sixty-five university students completed two trials on the O'Conner Tweezer Dexterity Test and completed questionnaires about perfectionism, self-esteem, and self-efficacy. As predicted, the adaptive perfectionists showed the greatest performance increases on the task as well as reporting higher self-esteem and self-efficacy than the other perfectionist styles. This study supports the perspective that perfectionism can be positive and negative.
\end{abstract}

Keywords: Perfectionism, Dexterity, Self-Esteem, Self-Efficacy, College Students

\section{Introduction}

The idea that perfectionism can be either a positive or negative personality trait stems from Alfred Adler's Individual Psychology [1]. Adler [1] believed that all humans have perfectionistic tendencies because "striving for perfection is innate" (p. 104). Adler also differentiated the normal aspects of perfectionism (e.g., striving to improve one's environment) from the abnormal aspects (e.g., striving to obtain an unattainable goal). Hamachek [2] had a similar view as Adler and believed that "normal" perfectionists strive to do their best and are not overly discouraged if they cannot do everything perfectly, whereas "neurotic" perfectionists strive for standards that cannot be met. Meeting academic and professional standards, as well as personal goals, is becoming increasing important. The competition to get into the best school, best job, and best position can be intense. Those who have high standards and who strive to do their best are the ones that may be able to be competitive academically and professionally. Conversely, those who set impossible goals for themselves may never experience satisfaction, which could lead to a variety of negative outcomes. Therefore, it is important to ascertain how both the positive and negative qualities of perfectionism affect performance and self-perceptions. The current study will investigate the relationship between perfectionist style and performance on a task when there are pre-existing and unattainable high standards. In addition, the effects of perfectionist style on self-esteem and self-efficacy will also be explored.

\subsection{Perfectionism}

Perfectionism, in general, is considered to have several key characteristics, such as high standards [3, 4], self-criticism [5], and a need for order [4, 6]. However, current research suggests that perfectionism is a multidimensional construct consisting of both positive and negative qualities. Researchers typically study perfectionism in two ways, either from an orientation perspective, or from an adaptive perspective. Hewitt and Flett [7] posit that perfectionism exists on three basic dimensions: self-oriented, other-oriented, and socially prescribed. Self-oriented perfectionism is having high standards and a motivation to obtain perfection for oneself. Conversely, socially prescribed perfectionism is the perception that one must obtain perfection to satisfy others. 
Lastly, other-oriented perfectionism is expecting others to be perfect. The second prevailing viewpoint on multidimensional perfectionism is the adaptive perspective. This perspective focuses on two dimensions of perfectionism, adaptive and maladaptive [4]. This perspective takes into consideration that not all aspects of perfectionism are negative.

Rice and associates [6] define an adaptive perfectionist as having high standards for oneself, a need for order, and "an unwillingness to procrastinate" (p. 311). In contrast, a maladaptive perfectionist is defined as being self-critical [5], having excessive concerns over mistakes [4, 8], and having high standards for oneself [3]. Interestingly, high standards are a defining feature of both the adaptive and maladaptive perfectionist. Aldea and Rice [3] state that the difference between the standards of the two perfectionist styles is the maladaptive perfectionist strives for standards that are "excessive and uncontrollable" (p. 505). There is current evidence to suggest that having excessive and unattainable standards for oneself leads to a variety of negative outcomes, such as stress [9], low academic achievement 4, 10], lower self-esteem [5, 11], and social functioning [10].

Research on perfectionism has generally focused on the performance of university students. Grade point average (GPA) has been used to measure both the actual performance and perceived performance in maladaptive and adaptive perfectionists. Empirical evidence suggests that maladaptive perfectionists have lower GPAs [10] and less satisfaction with their GPA [5]. This suggests that those who have excessive standards for themselves may be less effective in their school performance. High standards are the overarching theme among perfectionists in general and have therefore been of interest to researchers.

Stoeber and Eysenck [12] found that perfectionists who had higher standards took longer and marked more correct items wrong on a proofreading task than perfectionists with lower standards. Conversely, perfectionists who were more self-critical tended to miss errors in the proofreading task and marked fewer correct items wrong. However, when comparing participants who had higher strivings for perfectionism to those with lower strivings, high striving perfectionists spent more time on a task, self-reported a higher level of effort, and focused more on accuracy [13]. Those with low perfectionist strivings focused more on speed. This could suggest that while perfectionists tend to do better in situations where the accuracy of a task is more important than the speed, their high standards could lead to them second guessing themselves and reducing their effectiveness.

How perfectionists formulate their personal standards has also been of interest to researchers. In an experimental study, Kobori, Hayakawa, and Tanno [14] found that the level of self-oriented perfectionism was a significant predictor for raising standards for performance on a Stroop test. Moreover, participants who chose a higher goal performed marginally better than those who did not raise their standards. Kobori and colleagues suggested that some perfectionists might raise their standard for performance until the standard cannot be met because they believe they have to perform at the highest levels. Once the standards cannot be met, then perfectionists tend to be self-critical. Within the adaptive perspective of perfectionism, self-criticism is the key feature that separates the adaptive perfectionist from the maladaptive perfectionist. Maladaptive perfectionists have been found to set higher goals for themselves, have more academic problems, and have lower self-esteem than adaptive perfectionists [15]. How one sets personal standards and appraises his or her progress has been correlated with an individual's sense of self-esteem and self-efficacy.

\subsection{Self-Esteem and Self-Efficacy}

Self-esteem is generally defined as how much self-worth or respect a person has for him or herself. The position that perfectionist style is related to self-esteem has been supported in recent literature. In their study, Blankenstein and associates [11] found that perfectionists who could not meet their excessive personal standards had lower self-esteem. It was also suggested that self-esteem plays a role in how a person uses coping skills. For example, someone with high self-esteem may react to failure by actively changing techniques or learning a new skill in order to succeed in a similar task in the future. However, a person with low self-esteem may believe that they are unable to do anything to change future outcomes. In addition, Aldea and Rice [3] suggest that "notions of success and failure are more flexible" (p. 506) in the adaptive perfectionist. Because adaptive perfectionists are better able to appropriately assess positive and negative outcomes, they are more likely to have a higher self-esteem than maladaptive perfectionists. The ability to appropriately assess outcomes is important to an individual's perception of self-efficacy.

Self-efficacy can be defined as how one feels about his or her ability to cope and perform in a situation [16]. Bandura [16] further stated that self-efficacy not only affects how people cope in a situation but also how much time and effort they are willing to put into a task. Because it has been found that those with a high striving for perfectionism tend to put forth more effort and spend more time on a task [13], it could be suggested that perfectionists may have higher levels of self-efficacy. However, self-efficacy is also tied to achievement of goals; those who consistently meet their goals have higher levels of self-efficacy than those who seldom meet their goals [16]. Therefore, maladaptive perfectionists who are self-critical and often have goals that cannot be achieved may have lower levels of self-efficacy. Stoeber, Hutchfield, and Wood [17] found that striving for perfectionism was positively correlated with an increased level of self-efficacy. Conversely, self-criticism was negatively correlated with self-efficacy. Adaptive perfectionists, who tend to set attainable goals, may have higher levels of self-efficacy. 


\subsection{The Current Study}

Much of the experimental research on perfectionism has been based on the person-oriented perspective, which tends to focus on the negative personal and social aspects of perfectionism. In contrast, the adaptive perspective has been mostly supported through correlational studies, focusing on the positive aspects of perfectionism and how they relate to academic performance, self-esteem, and self-efficacy. Therefore, the purpose of the present study is to contribute experimental support for the adaptive perspective. In the current study, it is predicted that adaptive perfectionists will show a greater increase in performance than maladaptive perfectionists in both a situation with pre-existing standards and one without standards. It is predicted that adaptive perfectionists will score higher on measures of self-esteem and self-efficacy than maladaptive perfectionists. In addition, someone who is not classified into either of the two groups (a nonperfectionist) would seemingly not experience the positive or negative effects of perfectionism. Therefore, a fourth prediction in this study is that nonperfectionists will perform differently on a task and have different levels of self-esteem and self-efficacy than the adaptive and maladaptive perfectionists.

\section{Method}

\subsection{Participants}

Participants were recruited from psychology classes at a mid-sized public university in the southern United States. Students who chose to participate received class credit or extra credit. An alternative activity was provided for those students who chose not to participate. Participants were comprised of 65 students (53 woman, 9 men, 3 declined to answer). The mean age of the participants was 22.63 (SD = 5.56). The ethnic composition of the sample was $72.3 \%$ Caucasian, $16.9 \%$ African American, 4.6\% Hispanic, 3.1\% Asian, 3.0\% Other. The sample contained $4.6 \%$ freshman, $13.8 \%$ sophomores, $46.2 \%$ juniors, and $35.4 \%$ seniors. Participants were treated in accordance with the American Psychological Association's (APA) standards and guidelines [18].

\subsection{Materials}

The Almost Perfect Scale-Revised (APS-R) [19] was used to measure participant's perfectionist style. The APS-R has 23 items and uses a 7-point Likert type scale that ranges from 1-strongly disagree to 7-strongly agree. The APS-R has three subscales that are used to place people into perfectionist categories. The High Standards subscale and Order Subscale are used to differentiate perfectionists from nonperfectionists. Sample items include, "I have high standards for my performance at work or at school" and "I think things should be put away in their place." The Discrepancy subscale is used to classify perfectionists as being maladaptive or adaptive. A sample item from this subscale includes, "My best never seems to be good enough for me."

The Rosenberg Self-Esteem Inventory (RSE) [20] was used to measure participant's self-esteem levels. The RSE has 10 items and uses a 4-point scale that ranges from 1-strongly disagree to 4-strongly agree. Sample items include, "On a whole, I am satisfied with myself" and "I take a positive attitude towards myself".

The General Self-Efficacy Inventory (GSE) [21] was used to measure participants perceived level of self-efficacy in coping with both typical life events and unanticipated live events. The GSE is a 10-item measure and uses a 4-point scale that ranges from 1-not me at all to 4-exactly true. Sample items include, "I can always manage to solve difficult problems if I try hard enough" and "I am confident that I could deal efficiently with unexpected events."

A basic demographic survey was used to determine the age, sex, and race/ethnicity of the participants. In addition, this survey asked for participants' current college GPA.

The O'Connor Tweezer Dexterity Test (by Lafayette Instruments, Model \#12-3030) was used to measure task performance. This task requires participants to pick up a small metal pin (one inch long and 1/16 inch in diameter) with tweezers and then place the pin into a 1/16-inch hole in a board with 10 rows and 10 columns of holes. Performance was measured by how many metal pins participants could place into the board within one minute.

\subsection{Procedure}

The Participants volunteered for a laboratory experiment entitled "Hand-Eye Coordination and Task Performance." Participants came into the laboratory individually and were told that the study concerned hand-eye coordination in college students and were given a consent form to complete. Participants were randomly assigned to either a high standards condition or a condition without standards. For the high standards condition, participants were told that most people could place 30 metal pins into the board in a minute, rather than the established norm of 14-16. In the condition without standards, participants were not given information about a standard outcome. In both conditions, participants were allowed to practice the O'Connor Tweezer Dexterity test to become acclimated to both the test and the testing environment. In both conditions, participants had one minute to place as many pegs into the apparatus as they could. After the minute had passed, a count was taken and the results were verbally communicated to the participant. Participants then completed the task a second time and the results were again verbally communicated to the participant. Participants then received a packet of questionnaires, which included the APS-R, RSE, GSE, and the demographic survey. Participants in both conditions were then debriefed and told the true purpose of the study. 


\section{Results}

Participants were separated into groups based on the survey responses on the APS-R: nonperfectionists $(n=21)$, adaptive perfectionists $(\mathrm{n}=15)$, and maladaptive perfectionists $(n=29)$. The cut-off scores for perfectionist categories were based upon the suggestion of Rice and Ashby's [4] criteria analysis. First, perfectionists were differentiated from nonperfectionists using the cut-off score of 42 . Scores 42 and above indicated that the participant was a perfectionist and scores below 42 indicated that the participant was a nonperfectionists. Second, participants who were categorized as perfectionists by the High Standards subscale were classified as being adaptive or maladaptive perfectionists based upon their scores on the Discrepancy subscale. Scores 42 and above indicated that the participant was a maladaptive perfectionist and scores below 42 indicated that the participant was an adaptive perfectionist.

Mean change in task performance from time 1 to time 2, self-esteem, and self-efficacy scores were calculated for each group. Mean change from time 1 to time 2 was used as the main dependent variable for performance since it demonstrates participant motivation to change based on initial scores. A series of 2 (Condition: high standard or no standard) x 3 (Perfectionist Style: adaptive, maladaptive or nonperfectionist) analysis of variance (ANOVA) tests were conducted for change in task performance from time 1 to time 2, self-esteem, and self-efficacy.

The two-way ANOVA for change in task performance, measured as a change from trial 1 to trial 2 , indicated a marginally significant main effect for perfectionist style, $\mathrm{F}$ $(2,63)=2.95, \mathrm{p}=.06, \eta_{\mathrm{p}}{ }^{2}=.09$. Post hoc analysis using Tukey's HSD criterion indicated that there were marginally significant differences between the mean task performance scores between the adaptive perfectionist group $(\mathrm{M}=2.76$, $\mathrm{SD}=2.92)$ and the maladaptive perfectionist group $(\mathrm{M}$ $=.20, \mathrm{SD}=2.98), \mathrm{p}=.06$. There was no significant difference between the nonperfectionist group $(\mathrm{M}=1.90$, $\mathrm{SD}=4.36)$ and the adaptive $(\mathrm{p}=.66)$ and maladaptive perfectionist group $(p=.31)$. There was a marginally significant main effect for condition, $F(1,64)=3.43$, p $=.07, \eta_{\mathrm{p}}{ }^{2}=.06$. The mean task performance score for the high standards group $(\mathrm{M}=2.64, \mathrm{SD}=3.56)$ was higher than the no standards group $(\mathrm{M}=1.13, \mathrm{SD}=3.60)$. There was not an interaction effect between perfectionist style and condition on mean task performance, $\mathrm{F}(2,63)=.16, \mathrm{p}$ $=.85, \eta_{\mathrm{p}}{ }^{2}=.006$. See Fig. 1 for results.

The two-way ANOVA for self-esteem indicated a significant main effect of perfectionist style, $F(2,63)=$ 18.09, $\mathrm{p}<.001, \eta_{\mathrm{p}}{ }^{2}=.38$. Post hoc analysis using Tukey's HSD criterion indicated that there were significant differences between the mean self-esteem scores between

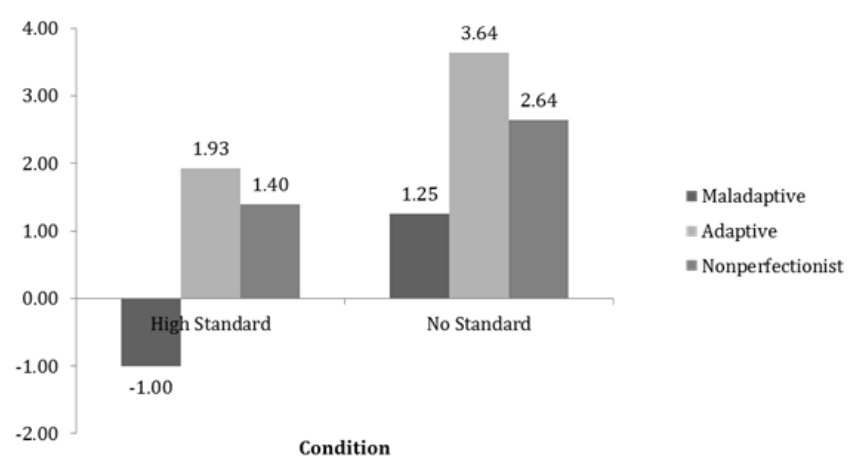

Figure 1. Mean difference between Trial 1 and Trial 2 for pegs placed in board during the O'Connor Dexterity Test by condition and perfectionist group.

the adaptive perfectionist group $(\mathrm{M}=2.66, \mathrm{SD}=.30)$ and the maladaptive perfectionist group $(\mathrm{M}=2.20, \mathrm{SD}=.36), \mathrm{p}$ $<.001$. There were also significant differences between the adaptive perfectionist group and the nonperfectionist group $(\mathrm{M}=2.11, \mathrm{SD}=.38), \mathrm{p}<.001$. There was no significant difference between the mean self-esteem scores between the maladaptive and nonperfectionists groups, $\mathrm{p}=.74$. There were no main effects for condition, $\mathrm{F}(1,64)=2.01$, $\mathrm{p}=.16, \eta_{\mathrm{p}}{ }^{2}=.03$, nor was there an interaction effect between perfectionist style and condition on mean self-esteem scores, $F(2,63)=.10, p=.90, \eta_{p}{ }^{2}=.004$. See Fig. 2 for results.

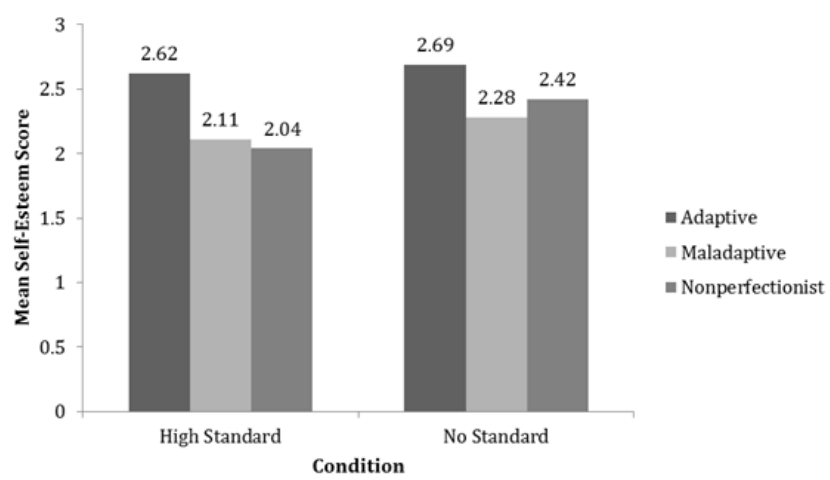

Figure 2. Mean self-esteem scores by condition and perfectionist group.

The two-way ANOVA for self-efficacy indicated a significant main effect of perfectionist style, $\mathrm{F}(2,63)=$ $8.25, \mathrm{p}=.001, \eta_{\mathrm{p}}{ }^{2}=.22$. Post hoc analysis using Tukey's HSD criterion indicated that there were significant differences between the mean self-efficacy scores between the adaptive perfectionist group $(\mathrm{M}=3.40, \mathrm{SD}=.39)$ and the nonperfectionist group $(\mathrm{M}=2.96, \mathrm{SD}=.33) \mathrm{p}<.001$. There was a marginally significant differences between the self-efficacy scores of the maladaptive perfectionist group $(\mathrm{M}=3.25, \mathrm{SD}=.42)$ and the nonperfectionist group, $\mathrm{p}$ $=.07$. There were no significant differences in the mean self-efficacy scores between the adaptive and maladaptive perfectionist group, $\mathrm{p}=.44$. There were no main effects for condition, $\mathrm{F}(1,64)=1.08, \mathrm{p}=.30, \eta_{\mathrm{p}}{ }^{2}=.02$, nor was there an interaction effect between perfectionist style and condition on mean self-efficacy scores, $F(2,63)=.49, p$ 
$=.62, \eta_{\mathrm{p}}^{2}=.02$. See Fig. 3 for results.

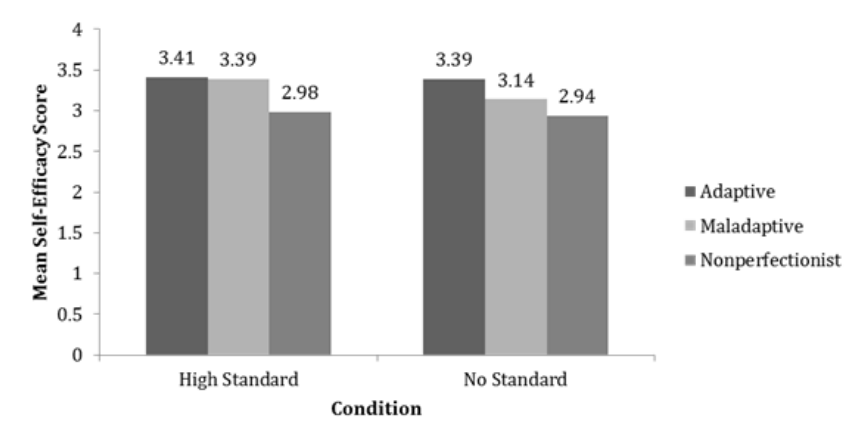

Figure 3. Mean self-efficacy scores by condition and perfectionist group

Additionally, GPA was positively correlated with the combined High Standards subscale and Order Subscale of the APS-R, $\mathrm{r}(63)=.37, \mathrm{p}<.001$, showing that perfectionists had higher GPAs. GPA was negatively correlated with the Discrepancy subscale of the APS-R, $r$ $(63)=-.13, p=.29$, showing GPA was not significantly different depending on whether your perfectionism was adaptive or maladaptive.

\section{Discussion}

The pattern of results of this study supported the hypotheses. The adaptive perfectionists did show a larger increase in performance (marginally significant difference) on the dexterity task than the maladaptive perfectionists in both the high standards and no standards condition, and the nonperfectionists scored between these two groups. It was assumed that because the O'Conner Tweezer Dexterity Test was a novel task for most participants, there would be an improvement in mean performance for all the groups after the first trial. Interestingly, the performance of maladaptive perfectionists in the high standards condition actually decreased. Hanchon [15], relating perfectionism to goal theory, found that maladaptive perfectionists had higher levels of performance goals (both approach and avoidance) while adaptive perfectionists had higher levels of mastery goals. In terms of the current study, it could be possible that maladaptive perfectionists could have focused more on meeting the "normal" standard or decided to give up after failing the first time. Adaptive perfectionists, while having high standards for themselves, may be more capable of focusing exclusively on a task when necessary.

Results also showed participants displayed a greater increase in performance in the high standards condition where they were instructed to do their best, compared to the no standards condition where this information was not provided. One reason for this finding is that the lack of knowledge of pre-existing standards may have allowed the participants in the no standards condition to focus exclusively on the task. Conversely, participants in the high standards condition may have realized after the first trial that they were failing to meet the "normal" standard of performance. In the subsequent trial, this may have led to the participants not trying as hard or becoming preoccupied with meeting the pre-existing standard.

The results of this study supported the hypothesis that adaptive perfectionists would score significantly higher on a measure of self-esteem than adaptive perfectionists. However, this study found only partial support for the hypothesis that nonperfectionists would differ from both the adaptive and maladaptive perfectionists. The self-esteem scores of nonperfectionists were only significantly different from that of the adaptive perfectionists. This finding is consistent with the results of Grzegorek and associates' [5] study that found that adaptive perfectionists scored significantly higher on the RSE measure than maladaptive perfectionists. The finding that the nonperfectionists had similar self-esteem scores to maladaptive perfectionists is also consistent with previous studies $[5,22]$.

Adaptive perfectionists may have higher self-esteem than maladaptive perfectionists because they tend to set goals for themselves that are achievable. The ability for the adaptive perfectionist to consistently meet goals may have a positive effect on their self-esteem. Conversely, maladaptive perfectionists have goals that are unattainable and excessive. Aldea and Rice [3] suggested that maladaptive perfectionists are unable to recognize ordinary success, and focus instead on any slight mistakes that might have occurred. The discrepancy between performance expectations and perceived actual performance may lead maladaptive perfectionists to have a more negative view about themselves and may contribute to their lower levels of self-esteem. Being aware of the negative effects that maladaptive perfectionism has on self-esteem may not be enough to help clients increase their self-esteem. One study found that participants who received feedback about their maladaptive perfectionism did not experience increases in their self-esteem levels two weeks later [23]. This suggests that perhaps it is not enough to increase self-esteem to know maladaptive behaviors and thoughts are unhealthy. Self-esteem may be changeable only when the person experiences consistent and positive outcomes from a change in behavior and/or perceptions of performance.

It is interesting that there were no difference between the self-esteem scores of the maladaptive perfectionists and nonperfectionists. Out of the three perfectionist groups, nonperfectionists had the lowest self-esteem scores. This suggests that the discrepancy between performance expectations and perceived actual performance is not the only factor affecting self-esteem. It may be that the lack of a goal to meet is also important. Rice and Ashby [4] suggest that the term "nonperfectionist" may not be an appropriate classification label when using the APS-R as groups are first distinguished by scores on the High Standards subscale. Many of the participants in the nonperfectionists group had high $(>42)$ discrepancy scores. This suggests that nonperfectionists may lack the ability to know how to successfully meet goals. This may also contribute to their lower levels of self-esteem.

The hypothesis that adaptive perfectionists would score 
higher than maladaptive perfectionists on a measure of self-efficacy was not fully supported, although adaptive perfectionists did report the highest self-efficacy scores overall. There was partial support for the hypothesis that nonperfectionists would score differently than both adaptive and maladaptive perfectionists. Nonperfectionists scored significantly lower on the GSE than adaptive perfectionists. One explanation for the similarity in the self-efficacy scores between the adaptive and maladaptive perfectionists is that both groups are willing to exert a great deal of effort to meet their goals. The results of this study suggest that even though maladaptive perfectionists are rarely able to meet their excessive goals, they still perceive themselves as able to cope and perform effectively.

There were some limitations to this study. First, participants were comprised of university students of which the majority $(68 \%)$ were classified as being perfectionists. The majority of the participants were Caucasian females. It is not known if these results will generalize to other, more diverse populations. Second, task performance was only measured across two trials. It is not known if further increases or decreases in performance would have continued in subsequent trials. Further, the presence of the researcher may have also affected performance especially in the high standards condition. Maladaptive perfectionists may have been more concerned about failing in front of a person rather than not meeting a particular standard. The APS-R was completed after completing the task and therefore, it is possible that the participants' perceived performance on the task could have affected how they responded to items. Specifically, depending on the condition, participants may have been primed to be more or less self-critical.

Future studies should focus on the actual performance of adaptive and maladaptive perfectionists. The results of the current study suggests there are differences in performance between the adaptive and maladaptive perfectionists. The self-criticism and concerns over mistakes may cause maladaptive perfectionists to be less effective and efficient. It would also be advantageous to study other populations besides university students. It could be possible that differences in performance would be clearer as there are often more costs and benefits associated with performance within an organization. Lastly, the nonperfectionist group and maladaptive perfectionist group were similar in performance and self-esteem. It may be advantageous to further define the term "nonperfectionist."

\section{Conclusions}

In conclusion, this study found that perfectionist style effects dexterity performance. In addition, perfectionist style is related to self-esteem and self-efficacy. In this study, self-esteem scores were significantly different depending on perfectionist style, which supports the idea that perfectionism is a multidimensional construct. The results of this research may prove useful to university counselors and academic advisors working with students who may have perfectionistic tendencies. Maladaptive perfectionists may need assistance in setting appropriate and manageable goals for themselves. Further, they may need to learn coping skills to help them react appropriately to the stressors and disappointments in life. Perfectionism is a personality trait, but it does not necessarily have to be a negative one. Helping people recognize and retain the positive aspects of perfectionism while ameliorating the negative effects may allow people to be more effective and productive throughout their lives.

\section{References}

[1] Ansbacher, H. L., \& Ansbacher, R. R. (Eds). (1991). The individual psychology of Alfred Adler. New York: Basic Books.

[2] Hamachek, D. E. (1978). Psychodynamics of normal and neurotic perfectionism. Psychology: A Journal of Human Behavior, 15(1), 27-33.

[3] Aldea, M. A., \& Rice, K. G. (2006). The role of emotional dysregulation in perfectionism and psychological distress. Journal of Counseling Psychology, 53(4), 498-510. doi:10.1037/0022-0167.53.4.498

[4] Rice, K. G., \& Ashby, J. S. (2007). An efficient method for classifying perfectionists. Journal of Counseling Psychology, 54(1), 72-85. doi:10.1037/0022-0167.54.1.72

[5] Grzegorek, J. L., Slaney, R. B., Franze, S., \& Rice, K. G. (2004). Self-criticism, dependency, self-esteem, and grade point average satisfaction among clusters of perfectionists and nonperfectionists. Journal of Counseling Psychology, 51(2), 192-200. doi: 10.1037/0022-0167.51.2.192

[6] Rice, K. G., Ashby, J. S., \& Slaney, R. B. (1998). Self-esteem as a mediator between perfectionism and depression: A structural equations analysis. Journal of Counseling Psychology, 45(3), 304-314. doi:10.1037/0022-0167.45.3.304

[7] Hewitt, P. L., \& Flett, G. L. (1991). Perfectionism in the self and social contexts: Conceptualization, assessment, and association with psychopathology. Journal of Personality and $\begin{array}{llll}\text { Social } & \text { Psychology, 60(3), }\end{array}$ doi:10.1037/0022-3514.60.3.456

[8] Hibbard, D. R., Davies, K. L. (2011). Perfectionism and psychological adjustment among college students: Does educational context matter? North American Journal of Psychology, 13(2), 187-200.

[9] Chang, E. C., Watkins, A., \& Banks, K. (2004). How adaptive and maladaptive perfectionism relate to positive and negative psychological functioning: Testing a stress-mediation model in black and white female college students. Journal of Counseling Psychology, 51(1), 93-102. doi:10.1037/0022-0167.51.1.93

[10] Gilman, R., \& Ashby, J. S. (2003). Multidimensional perfectionism in a sample of middle school students: An exploratory investigation. Psychology in the Schools, 40(6), 677-689. doi:10.1002/pits.10125

[11] Blankstein, K. R., Dunkley, D. M., \& Wilson, J. (2008). Evaluative concerns and personal standards perfectionism: 
Self-esteem as a mediator and moderator of relations with personal and academic needs and estimated GPA. Current Psychology, 27(1), 29-61. doi:10.1007/s12144-008-9022

[12] Stoeber, J., \& Eysenck, M. W. (2008). Perfectionism and efficiency: Accuracy, response bias, and invested time in proof-reading performance. Journal of Research in Personality, 42(6), 1673-1678. doi:10.1016/j.jrp.2008.08.001

[13] Stoeber, J., Chesterman, D., \& Tarn, T. (2010). Perfectionism and task performance: Time on task mediates the perfectionistic strivings-performance relationship. Personality and Individual Differences, 48(4), 458-462. doi:10.1016/j.paid.2009.11.021

[14] Kobori, O., Hayakawa, M., \& Tanno, Y. (2009). Do perfectionists raise their standards after success? An experimental examination of the revaluation of standard setting in perfectionism. Journal of Behavior Therapy and Experimental Psychiatry, 40(4), 515-521. doi:10.1016/j.jbtep.2009.07.003

[15] Hanchon, T. A. (2010). The relations between perfectionism and achievement goals. Personality and Individual Differences, 49, 885-890. doi:10.1016/j.paid.2010.07.023

[16] Bandura, A. (1977). Social learning theory. Englewood Cliffs, NJ: Prentice-Hall.

[17] Stoeber, J., Hutchfield, J., \& Wood, K. V. (2008). Perfectionism, self-efficacy, and aspiration level: Differential effects of perfectionistic striving and self-criticism after success and failure. Personality and Individual Differences,
45(4), 323-327. doi:10.1016/j.paid.2008.04.021

[18] American Psychological Association (2002). Ethical principles of psychologists and code of conduct. American Psychologist, 57, 1060-1073.

[19] Slaney, R. B., Rice, K. G., Mobley, M., Trippi, J., \& Ashby, J. S. (2001). The revised Almost Perfect Scale. Measurement and Evaluation in Counseling and Development, 34, $130-145$.

[20] Rosenberg, M. (1965). Society and the adolescent self-image. Princeton, NJ: Princeton University Press.

[21] Schwarzer, R., \& Jerusalem, M. (1995). Generalized self-efficacy scale. In J. Weinman, S. Wright, \& M. Johnston (Eds.), Measures in health psychology: A user's portfolio. Causal and control beliefs (pp. 35-37). Windsor, UK: NFER Nelson.

[22] Rice, K. G., Ashby, J. S., \& Gilman, R. (2011). Classifying adolescent perfectionists. Psychological Assessment, 23(3), 563-577. doi:10.1037/a0022482

[23] Aldea, M. A., Rice, K. G., Gormley, B., \& Rojas, A. (2010). Telling perfectionists about their perfectionism: Effects of providing feedback on emotional reactivity and psychological symptoms. Behaviour Research and Therapy, 48(12), 1194-1203. doi:10.1016/j.brat.2010.09.003. 